\title{
Pancreatic Intraductal Papillary-Mucinous Neoplasm with Intermediate Grade Dysplasia
}

National Cancer Institute

\section{Source}

National Cancer Institute. Pancreatic Intraductal Papillary-Mucinous Neoplasm with

Intermediate Grade Dysplasia. NCI Thesaurus. Code C5719.

A non-invasive pancreatic intraductal papillary mucinous neoplasm characterized by the presence of neoplastic epithelial cells that exhibit nuclear stratification, loss of polarity, and crowding. There is moderate nuclear hyperchromasia. 